\title{
Design of 3D force perception system of surgical robots based on Fiber Bragg Grating
}

\author{
Haodong Wang ${ }^{1}$, Weidong Wang ${ }^{1}$, Zhijiang $\mathrm{Du}^{1}$, and Yongzhuo Gao ${ }^{1, *}$ \\ ${ }^{1}$ No. 92, Xidazhi Street, Nangang District, Harbin City, Heilongjiang Province, China
}

\begin{abstract}
Surgical robots have been widely researched due to their features of accurate positioning, no jitter, high precision, and low error rate under certain tasks. However, it is generally relatively slow to the feedback generated by force, deformation, or sudden and arbitrary impact during operation. Besides, the feedback is always provided through a vision that cannot meet the actual operation requirements for doctors. The customized design and integration of force perception functions on different robots have become one of the research hotspots of surgical robotics-worldwide recently. A force perception sensor for surgical robots based on Fiber Bragg grating (FBG) is proposed in this paper. It can be used to measure three-dimensional force. The experimental parameters are utilized to calibrate the model through the least square method. A four DoFs experimental platform is constructed. The system errors of the sensor involved are evaluated. The effectiveness of the proposed algorithm can be proved by the experimental results
\end{abstract}

\section{Introduction}

Compared with traditional open surgery, minimally invasive surgical robot technology can effectively reduce the risk of surgical infection and the probability of complications[1,2]. It has the advantages of less surgical trauma, light pain, less blood loss during the operation, and quick recovery after surgery. Its excellent medical effects have been widely recognized by the industry and favored by patients[3]. Currently, minimally invasive surgical robots still have many shortcomings. For example, its ability to perceive external forces is poor[4].

In the past ten years, with the popularization and application of minimally invasive surgical robots, many research institutions have successively launched the design and development of surgical robot force perception systems. M. Sorli et al. designed a six DoFs Stewart force sensor based on the famous Gough-Stewart parallel platform and analyzed its static and dynamic performance[5]. Mathias Stefan Müller et al. proposed a Stewart sensor with FBG instead of a supporting beam structure[6]. Robert Haslinger et al. integrated the FBG into the support beam of the Stewart sensor to obtain the strain of each joint[7]. The integration of FBG not only solved the biocompatibility problem but also improved the integration and layout space of the sensor. The sensitivity of FBG can resolve the sensor

\footnotetext{
* Corresponding author: gaoyongzhuo@hit.edu.cn
} 
higher. Xue et al. designed a flexible joint with multiple cantilever beams, which is manufactured by $3 \mathrm{D}$ printing technology[8]. The raw material is photosensitive resin C-UV 9400. The FBG sensor is fixed to the groove of the beam through glue.

Among these research methods and sensing methods, the fiber Bragg grating sensor (FBG) has the advantages of anti-electromagnetic interference, high-temperature resistance, small size, corrosion resistance, etc[9]. Due to the difference in surgical background and medical use, the characteristics, indicators, and performance of the force perception system designed are also different. A force sensor based on FBG is designed in this paper. The three-dimensional force is decoupled. The sensor can be utilized to realize the perception of three-dimensional force. The structure design is introduced in Section 2. The force perception model is constructed in Section 3. Experimental verification is performed in Section 4. The conclusion is introduced in Section 5.

\section{Structure analysis of three-dimensional force sensor}

The proposed three-dimensional force sensor is composed of four beams, which enable the deformation of the robot effector to be concentrated on the specific structure of the flexible joint. Theoretically, the acquisition of external force space vector information can be achieved with three sensors. However, in order to ensure the accuracy and reliability of the sensing system, a sensor is designed which includes the flexible joints with four FBGs and a sensing structure with a central FBG. The two ends of the flexible joint are designed with axial and radial connection and fixing mechanisms. The cross-sectional connected domain of the material is larger than the sum of the cross-sectional areas of the four elastic beams to ensure that the flexibility is concentrated at the flexible joints. The design purpose of the front cross beam mechanism of the flexible joint is to make the force transmitted from the robot wrist more concentratedly act on the four elastic beams.
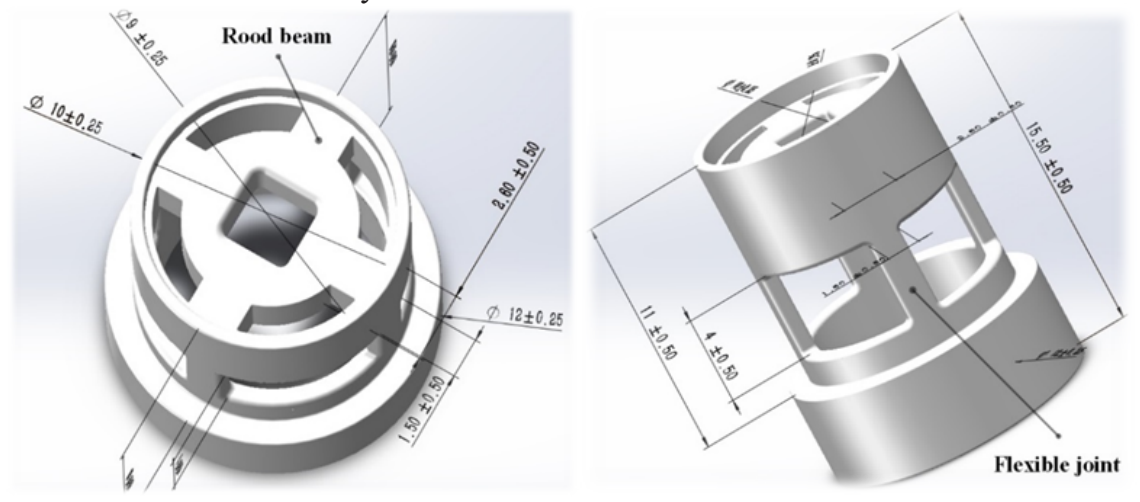

Fig. 1. Schematic diagram of the force sensor.

The force of the surgical robot is transmitted to the center of the wrist through the end effector. Therefore, the force of the end of the surgical robot is equivalent to the center of the wrist in the actual modeling process. The designed force sensing system is equipped with wrist joints to reduce the cost of the experimental process, before proving the performance of the sensing system. The micro-end sensing structure equipped with 4 FBGs is shown in Fig.2. 


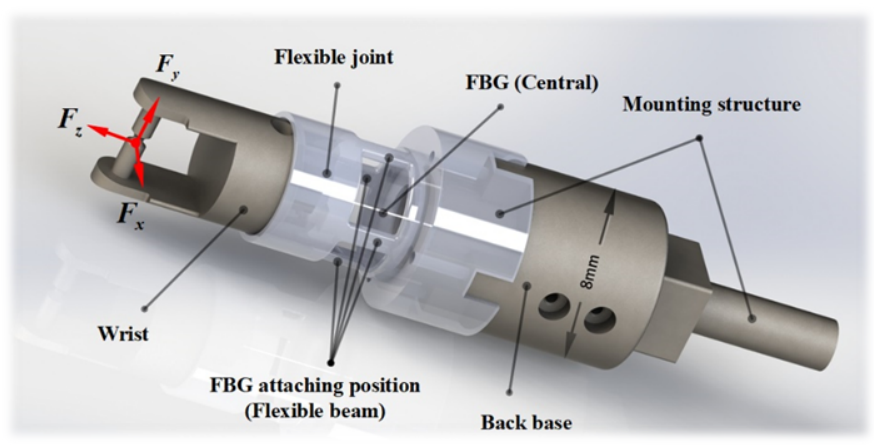

Fig. 2. Schematic diagram of micro-end sensing structure equipped with 4 FBGs.

\section{Construction of force perception model}

The designed force sensing system requires a total of five FBG. The FBG located at the neutral axis is used to directly sense the axial force of the flexible joint, and the other four FBGs located at the elastic beam are used to sense the lateral force. The elastic beam strain caused by the external force is the effect of coupling. Therefore, before calculating the lateral force through the measurement data, the measurement results of the remaining four FBGs need to be decoupled by the wavelength change of the center FBG.

Theoretically, the model of the designed force sensing system can be expressed as follows:

$$
\boldsymbol{\Delta} \boldsymbol{\lambda}=\boldsymbol{C} \cdot \boldsymbol{F} \Rightarrow\left[\begin{array}{c}
\boldsymbol{\Delta} \lambda_{A} \\
\boldsymbol{\Delta} \lambda_{B} \\
\boldsymbol{\Delta} \lambda_{C} \\
\boldsymbol{\Delta} \lambda_{D} \\
\boldsymbol{\Delta} \lambda_{O}
\end{array}\right]_{5 \times n}=\boldsymbol{C}_{5 \times 3} \cdot\left[\begin{array}{c}
\boldsymbol{F}_{\boldsymbol{x}} \\
\boldsymbol{F}_{\boldsymbol{y}} \\
\boldsymbol{F}_{z}
\end{array}\right]_{3 \times n}
$$

where $\boldsymbol{F}$ represents the input signal of the sensor system; $\Delta \boldsymbol{\lambda}$ represents the output of the sensor system; $\boldsymbol{C}$ represents the characteristic matrix obtained by calibration of the sensor system in the experiment.

Before calibrating the system using the least square method, the equation (1) can be transposed as follows:

$$
\boldsymbol{F}^{T} \cdot \boldsymbol{C}^{T}=\Delta \lambda^{T}
$$

$C$ can be obtained through the two-norm minimum solution of the equation (2).

$$
\min _{\boldsymbol{C}}\left\|\boldsymbol{F}^{T} \boldsymbol{C}^{T}-\boldsymbol{\Delta} \boldsymbol{\lambda}^{T}\right\|_{2}=\boldsymbol{F}^{T \dagger} \cdot \Delta \boldsymbol{\lambda}^{T}
$$

where $\boldsymbol{F}^{T \dagger}=\left(\boldsymbol{F} \cdot \boldsymbol{F}^{\boldsymbol{T}}\right)^{-1} \cdot \boldsymbol{F}$ represents the Moore-Penrose generalized inverse matrix of the system input matrix.

\section{Experimental verification}

The experimental platform is composed of a four-degree-of-freedoms(DOFs) platform, a robot wrist joint integrated with an FBG force sensing system, an FBG demodulator, a high-precision electronic balance, a C-shaped weight, a vertical lanyard fixing mechanism, and a computer, as shown in Fig.3. The wrist joint of the robot with the FBG force sensor is fixedly connected with the 4 DOFs platform, and the center position of the joint is 
guaranteed to coincide with the remote center mechanism (RCM) of the 4 DOFs platform. A rope is tied to the center of the joint, and the end of the rope is tied to a hanging plate. A high-precision electronic balance is placed under the hanging plate as a reference for force measurement. The adjustment of the joint force can be realized by changing the weight on the hanging plate, or by adjusting the height of the motion platform in the Z-axis. The actual value of the force $\boldsymbol{F}$ on the joint is obtained as

$$
\boldsymbol{F}=M g-m g=(M-m) g
$$

where $M$ is the mass of the hanging plate and the weight, $m$ is the reading of the electronic balance.

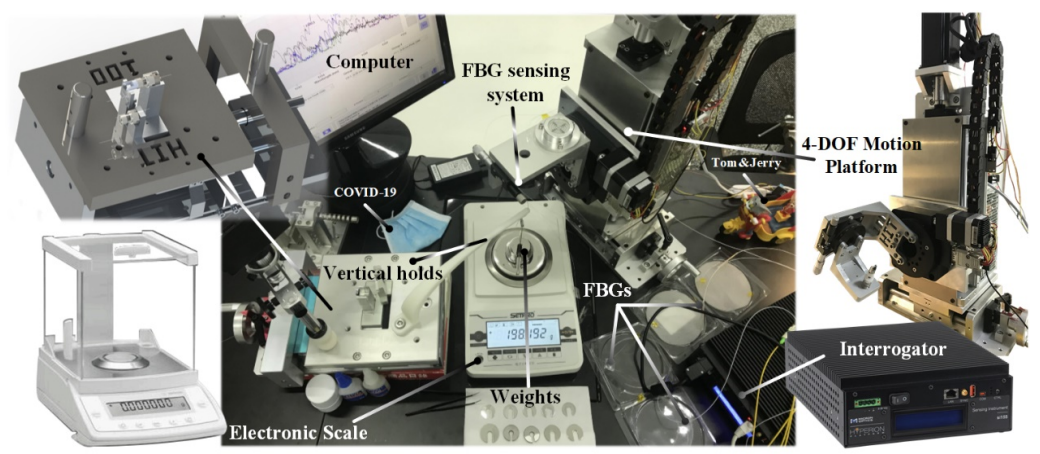

Fig. 3. FBG force perception system experiment.

There are four signal channels in the si-155 demodulator. Three adjacent edge FBG and central FBG channel signal changes have been detected in actual experiments.

The calibration experiment of the FBG force sensing system is carried out under constant temperature conditions. The steps are as follows.

The adjustment range of turntable 1 is set to $\left[0, \alpha_{\max }\right]$. The adjustment range of turntable 2 is set to $\left[0, \beta_{\max }\right]$, as shown in Fig.4. The external force is assumed to be always vertical. The external force at the center of the current wrist can be calculated according to equation (4). According to the specific wrist posture, the three-dimensional force received by the wrist can be obtained as follows:

$$
\boldsymbol{F}=\left[\begin{array}{lll}
F_{x} & F_{y} & F_{z}
\end{array}\right]=\left[\begin{array}{lll}
F \cos \alpha \sin \beta & F \sin \alpha & F \cos \alpha \cos \beta
\end{array}\right]
$$

where $\alpha$ and $\beta$ refer to the relative rotation angles of the turntables, respectively.

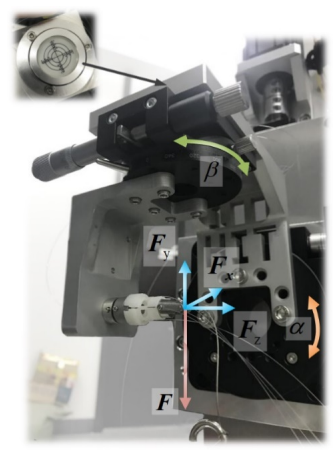

Fig. 4. The calculation diagram of the three-dimensional force.

The calibration matrix of the sensing system in the sense of orthogonality can be obtained through formula (3). The FBG sensing matrix in the $\beta=0^{\circ}$ state can be obtained as 


$$
\left[\begin{array}{l}
\Delta \lambda_{1} \\
\Delta \lambda_{2} \\
\Delta \lambda_{3} \\
\Delta \lambda_{4}
\end{array}\right]_{4 \times n}^{\beta=0}=\left[\begin{array}{ccc}
0 & -0.413 & 0.067 \\
0 & 0.016 & 0.067 \\
0 & 0.484 & 0.020 \\
0 & 0.004 & 0.005
\end{array}\right] \cdot\left[\begin{array}{l}
\boldsymbol{F}_{\boldsymbol{x}} \\
\boldsymbol{F}_{\boldsymbol{y}} \\
\boldsymbol{F}_{z}
\end{array}\right]_{3 \times n}^{\beta=0}
$$

The accuracy of the si-155 demodulator is $1 \mathrm{pm}$. The resolutions of $F_{y}$ and $F_{z}$ can be obtained through model (6). The resolutions of $F_{y}$ and $F_{z}$ are $1.006 \mathrm{mN}$ and $18.854 \mathrm{mN}$, respectively. Therefore, the resolution of the system's total input external force is 18.881 $\mathrm{mN}$. The comparison between the theoretical and experimental results of external forces is shown in Fig.5.
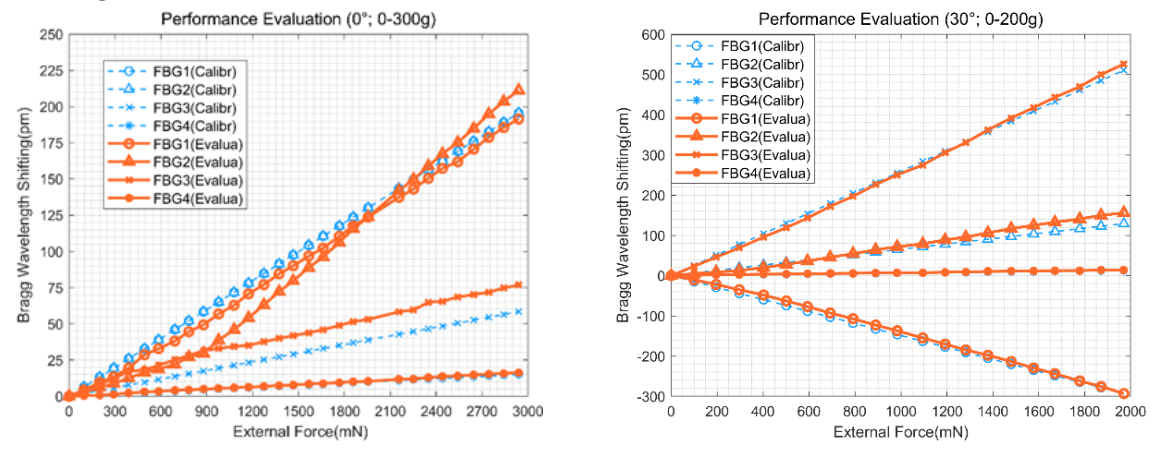

(a) $\boldsymbol{\alpha}=0^{\circ}, \boldsymbol{\beta}=0$

(b) $\boldsymbol{\alpha}=30^{\circ}, \boldsymbol{\beta}=0^{\circ}$
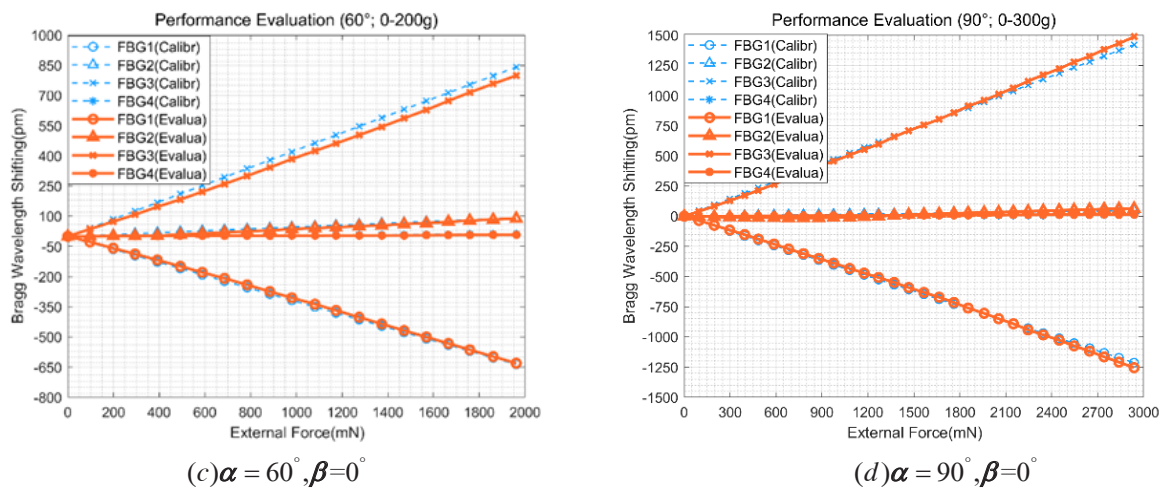

Fig. 5. The comparison results.

The maximum input nonlinear errors of the four FBG channels are $40.956 \mathrm{pm}$, $28.747 \mathrm{pm}, 65.060 \mathrm{pm}, 4.582 \mathrm{pm}$, respectively. The nonlinear error of $F_{y}$ and $F_{z}$ can be obtained by inversely solving equation (3) as

$$
\boldsymbol{F}_{\text {Error }}=\boldsymbol{C}^{\dagger} \cdot \Delta \lambda_{\text {Error }}
$$

The non-linear errors of $F_{y}$ and $F_{z}$ are $0.069 \mathrm{~N}$ and $0.767 \mathrm{~N}$, respectively. Therefore the non-linear error of the resultant force is $0.770 \mathrm{~N}$.

\section{Conclusion}

In traditional surgery, the surgeon can feel the force through touch. However, there is a lack of force perception in surgical robots. Based on FBG, a three-dimensional force sensor is designed which can be used in surgical robots. The experimental parameters are calibrated 
based on the least square method. Based on the experimental platform, the proposed force sensors were evaluated. The experimental results show that the non-linear error of the resultant force is $0.770 \mathrm{~N}$. The experimental results show that the designed sensor can be used in the field of surgical robots, and future work will focus on the system integration of force sensors.

This research was supported in part by the National Natural Science Foundation of China under Grant No.61673139 and Grant No. U1813213.

\section{References}

1. Wang, H., Wang, X., Yang, W. et al. Design and Kinematic Modeling of a Notch Continuum Manipulator for Laryngeal Surgery. Int. J. Control Autom. Syst. 18, 11 (2020).

2. Wang, H., Wang, X., Yang, W. et al. Construction of Controller Model of Notch Continuum Manipulator for Laryngeal Surgery Based on Hybrid Method. IEEE/ASME Transactions on Mechatronics (Early Access Article) (2020)

3. A. Gao, R. J. Murphy, H. Liu, I. I. Iordachita and M. Armand. Mechanical Model of Dexterous Continuum Manipulators With Compliant Joints and Tendon/External Force Interactions. IEEE/ASME Transactions on Mechatronics, 22, 1, (2017).

4. W. Hong, L. Xie, J. Liu, Y. Sun, K. Li and H. Wang. Development of a Novel Continuum Robotic System for Maxillary Sinus Surgery. IEEE/ASME Transactions on Mechatronics, 23, 3, (2018).

5. Sorli M , Pastorelli S . Six-axis reticulated structure force/torque sensor with adaptable performances[J]. Mechatronics, 5, 6 (1995).

6. Müller, Mathias Stefan, Hoffmann L, Christopher Buck T, et al. Fiber Bragg GratingBased Force-Torque Sensor with Six Degrees of Freedom. International Journal of Optomechatronics, 3, 3 (2009).

7. Haslinger R, Leyendecker P, Seibold U . A Fiberoptic Force-Torque-Sensor for Minimally Invasive Robotic Surgery// ICRA 2013. IEEE, 2013.

8. Xue R, Ren Bingyin, Huang Jiaqing, et al. Design and Evaluation of FBG-Based Tension Sensor in Laparoscope Surgical Robots. Sensors, 18, 7, (2018).

9. A. Gao, Y. Zhou, L. Cao, Z. Wang and H. Liu. Fiber Bragg Grating-Based Triaxial Force Sensor With Parallel Flexure Hinges. IEEE Transactions on Industrial Electronics, 65, 10, (2018). 